\title{
Variación fenotípica y genética en el tunicado Pyura praeputialis (Heller, 1878) en el área norte de la Bahía de Antofagasta, Chile
}

\author{
Phenotypic and genetic variation in tunicate Pyura praeputialis (Heller, 1878) in the \\ northern sac of the Antofagasta Bay
}

MARCELA ASTORGA ${ }^{1}$, RICARDO GUIÑEZ ${ }^{2}$, JUAN C. ORTIZ ${ }^{3} \&$ JUAN C. CASTILLA $^{2}$

\begin{abstract}
${ }^{1}$ Programa Doctorado en Ciencias Biológicas-Área Zoología, Universidad de Concepción, Casilla 160-C, Concepción, Chile; e-mail: mastorga@udec.cl

${ }^{2}$ Centro de Estudios Avanzados en Ecología \& Biodiversidad, Facultad de Ciencias Biológicas, Pontificia Universidad Católica de Chile, Casilla 114-D, Santiago, Chile; e-mail:rguinez@genes.bio.puc.cl; jcastill@genes.bio.puc.cl

${ }^{3}$ Departamento de Zoología, Facultad de Ciencias Naturales y Oceanográficas, Universidad de Concepción, Casilla 160-C, Concepción, Chile; e-mail: jortiz@udec.cl
\end{abstract}

\begin{abstract}
RESUMEN
La morfología de los "piures" (Urochordata: Tunicata) es altamente variable, mostrando diferencias hábitat-específicas con una base intra- o interespecífica, lo cual dificulta la comprensión de sus significados taxonómicos, ecológicos y evolutivos. En el presente trabajo se caracterizó genéticamente, mediante polimorfismos de isoenzimas, a tres morfotipos de piures: achatados, intermedios y alargados, observados en poblaciones intermareales del "piure de Antofagasta" Pyura praeputialis. El estudio se realizó en tres localidades que se distribuyen en un gradiente de exposición al oleaje en el extremo norte de la Bahía de Antofagasta, Chile. Se analizó un total de 17 loci isoenzimáticos, a través de electroforesis en gel de almidón, de los cuales tres fueron polimórficos: IDH-1, MDH-1 y PGM-1. Los resultados sugieren diferencias morfológicas pero no genéticas entre los morfotipos. Sin embargo, las localidades difieren significativamente en su estructura genética. Se compara los resultados en relación a las respuestas observadas para morfotipos semejantes en Australia, sobre la base de que P. praeputialis habría colonizado recientemente la Bahía de Antofagasta. Se concluye que las diferencias entre los morfotipos en Antofagasta tienen una base intraespecífica y en Australia interespecífica y se discute las hipótesis que podrían explicar las diferencias genéticas y fenotípicas observadas.
\end{abstract}

Palabras clave: piure, Pyura praeputialis, morfotipos, exposición al oleaje, isoenzimas, electroforesis.

\begin{abstract}
The morphology of "sea squirts" (Urochordata: Tunicata) is highly variable and is expressed in habitat-specific differences intra- and interspecific variation pattern that had hindered the interpretation of such differences as well as their taxonomical, ecological or evolutionary meaning. Here we describe the population genetic structure of three morphotypes (squat, intermediate and tall "sea squirt") observed in populations of the "piure de Antofagasta", Pyura praeputialis by enzyme electrophoresis. The study was carried out in three localities in the northern sac of the Bay of Antofagasta, Chile, and distributed along a wave exposure gradient. A total of 17 enzymatic loci were assayed by horizontal starch-gel electrophoresis, three of which (IDH-1, MDH-1 and PGM-1) were polymorphic. The results showed significant genetic differences among localities, but not among morphotypes. These results are contrasted with similar studies conducted on the same species in Australia, on the base that P. praeputialis has recently colonized Antofagasta from Australia. It is suggested that the differences among the morphotypes from Antofagasta are intraspecifics and those from Australia are interspecifics. The possible explanations for the genetic and morphological differences among localities are discussed.
\end{abstract}

Key words: sea squirts, Pyura praeputialis, wave exposure, morphotypes, isozymes, electrophoresis.

\section{INTRODUCCIÓN}

La comprensión de la variación morfológica intraespecífica tiene importancia ecológico-evolutiva, especialmente si es de naturaleza clinal y de micro y meso escala geográfica (Johanesson et al. 1993). Los análisis genéticos que utilizan marcadores moleculares o metodologías genéticocuantitativas pueden permitir discriminar si la variabilidad morfológica corresponde a la expresión de diferencias interespecíficas crípticas, representadas por la presencia de dos o más especies, o a la expresión de la variación ecotípica intraespecífica modulada genética o ambien- 
talmente (e.g., Via \& Lande 1985, Stearns 1989, Johanesson \& Johanesson 1990, Johanesson et al. 1993, Scheiner 1993, Dalby 1997a, 1997b, Soler et al. 2000). En efecto, la utilización de marcadores moleculares en organismos marinos ha permitido determinar la presencia tanto de especies crípticas previamente no detectadas (Solé-Cava \& Thorpe 1986, Degnan \& Lavin 1995, Dalby 1997a, Soler et al. 2000), como de razas o subespecies (Flowerdew \& Crisp 1975, Dando \& Southward 1981). En otros casos, sobre la base de observaciones a micro y mesoescala espacial, manipulaciones experimentales o traslado de individuos a distintas condiciones ambientales, se ha podido demostrar que estas diferencias son mantenidas por procesos selectivos (Achituv \& Mizrahi 1987, Johanesson et al. 1993), o por modulaciones ambientales (Foster 1979, 1980, Lively 1986, Yund 1991, West et al. 1993, De Wolf et al. 1997, Bertness et al. 1998, Sarda et al. 1998, Leonard et al. 1999). En consecuencia, para una adecuada interpretación ecológica o evolutiva de la diferenciación morfológica intraespecífica, es necesario estudiar sus componentes genéticos y ambientales.

Pyura praeputialis (Heller, 1878) es un tunicado sésil de la familia Pyuridae que se distribuye en la costa oriental de Australia y en Chile sólo se presenta en la Bahía de Antofagasta (Castilla et al. 2000). Los patrones de evolución de los haplotipos del gen mitocondrial Citocromo Oxidasa I y su extremadamente bajo porcentaje de divergencia genética $(0,4 \%$ en promedio) entre especímenes de Australia y Chile, sugieren que la especie habría colonizado Antofagasta recientemente desde Australia (Castilla \& Guiñez 2000, Castilla et al. en prensa a). En Australia, existen dos morfotipos contrastantes de piures ("achatados" y "alargados") que inicialmente fueron considerados como formas ambientalmente moduladas (Kott 1952, 1976). Los piures achatados son de coloración amarilla y están restringidos a zonas estuarinas protegidas del oleaje, mientras que los piures alargados son de color café y de más amplia distribución geográfica, siendo frecuentes en roqueríos expuestos al oleaje (Dalby 1997a, 1997b). Sin embargo, la evidencia de marcadores aloenzimáticos y de experimentos de traslados recíprocos de individuos entre localidades sugiere que ambos morfotipos no son modulados ambientalmente y que corresponderían a dos especies distintas (Killingly 1976, Dalby 1997a, 1997b).

Debido a que, en el área norte de la Bahía de Antofagasta se ha observado morfotipos alargados y achatados de color café en $P$. praeputialis (Fig. 2), con una mayor proporción de los últimos en sitios de mayor exposición al oleaje, se pretende estudiar, (i) si existen diferencias genéticas aloenzimáticas entre los morfotipos de Antofagasta similares a las reportadas entre los morfotipos australianos, (ii) cómo se expresan las diferencias morfológicas y genéticas en un gradiente de intensidad del oleaje en Antofagasta, y (iii) sus implicancias ecológicas-evolutivas, en particular por el estatus de $P$. praeputialis como colonizador reciente en la Bahía de Antofagasta.

\section{MATERIALES Y MÉTODOS}

El trabajo se realizó en tres localidades del extremo norte de la Bahía de Antofagasta: El Edén $\left(23^{\circ}\right.$ $\left.32^{\prime} \mathrm{S}, 70^{\circ} 32^{\prime} \mathrm{O}\right)$, Las Conchillas $\left(23^{\circ} 31^{\prime} \mathrm{S}, 70^{\circ}\right.$ $32^{\prime}$ O) y La Rinconada ( $23^{\circ} 28^{\prime} \mathrm{S}, 70^{\circ} 30^{\prime} \mathrm{O}$ ) (Fig. 1). La distancia entre las localidades extremas El Edén y La Rinconada es de aproximadamente 10 km de línea de costa. Las localidades se ubican en un gradiente de exposición decreciente al oleaje: El Edén-Las Conchillas-La Rinconada. Para determinar la velocidad máxima del oleaje se usó cuatro dinamómetros por localidad instalados en el intermareal rocoso inferior siguiendo a Castilla et al. (1998).

Entre marzo y septiembre de 1999, se hizo un muestreo al azar de un total de 193 piures de $P$. praeputialis desde la franja intermareal rocosa inferior (Castilla 1981, 1998), para las tres localidades: El Edén $(\mathrm{n}=79)$, Las Conchillas $(\mathrm{n}=76)$ y La Rinconada $(\mathrm{n}=38)$. Para cada individuo se registró si se encontraba solitario o agregado. Los piures se trasladaron vivos a acuarios y se les midió tres variables gravimétricas (peso total, peso del cuerpo, y peso de la túnica) y cinco variables morfométricas (altura máxima, ancho superior paralelo y perpendicular a los sifones, ancho inferior a un $\mathrm{cm}$. de la base paralelo y perpendicular a los sifones; Fig. 2). Adicionalmente, se usó las variables morfométricas para estimar el volumen bajo la presunción de que la forma de los piures corresponde a un tronco de cono elipsoide recto (Fig. 2). El cuerpo de cada animal (sin túnica) se mantuvo en congelador a $84{ }^{\circ} \mathrm{C}$ hasta el momento de su análisis electroforético. Para establecer diferencias entre morfotipos y localidades para las variables de volumen y pesos del cuerpo y de la túnica se usó análisis univariado (ANDEVA) y multivariado (ANMUDEVA) de varianza con análisis canónico de dos vías con interacción y pruebas a posteriori de Tukey-HSD (Sokal \& Rohlf 1981), considerando como factores fijos la localidad y el morfotipo. 
La altura máxima ajustada de cada individuo “i”, $Y_{i}$, estandarizada en unidades de desviación estándar (DE), se utilizó para segregar a los individuos en los dos morfotipos extremos: achatados $\left(\mathrm{Y}_{\mathrm{i}}<\mathrm{Y}-0,5 \mathrm{DE}\right)$ y alargados $\left(\mathrm{Y}_{\mathrm{i}}>\mathrm{Y}+0,5 \mathrm{DE}\right)$, donde $\mathrm{Y}$ es la altura máxima ajustada promedio. Se incluyó también un tercer morfotipo denominado como intermedio, tal que $\left(\mathrm{Y}-0.5 \mathrm{DE} \leq \mathrm{Y}_{\mathrm{i}} \leq\right.$ $\mathrm{Y}+0,5 \mathrm{DE})$. Los morfotipos intermedios también han sido descritos como variantes intermedios en Australia (Kott 1952, 1976), pero no han sido estudiados previamente. De esta forma, cada morfotipo corresponde teóricamente a un $33 \%$ de la población total. La altura máxima ajustada se obtuvo siguiendo a Packard \& Boardman (1988) y Castilla et al. (2000), para lo cual se usó la regresión lineal ajustada (Modelo II: funcional, Sokal \& Rohlf 1981) del ancho máximo superior 1 (AM1) sobre la altura máxima (HM) para cada localidad y se ajustó cada valor a un ancho máximo promedio de $74,36 \mathrm{~mm}$.

Se ensayaron 13 enzimas solubles usando electroforesis horizontal en gel de almidón (Harris \& Hopkinson 1976, Whitmore 1990), lo que permitió resolver 17 loci isoenzimáticos (Tabla 1), tres de los cuales (PGM-1, IDH-1 y MDH-1) fueron polimórficos al criterio del $99 \%$. Este resultado confirmó datos previos obtenidos en piures de distintas localidades de la Bahía de Antofagasta sobre la base de más de 30 individuos por loci (M. Astorga resultados no publicados), por lo cual, en este trabajo, los loci monomórficos fueron analizados en sólo 10 individuos por morfotipo, con el objetivo de confirmar el estado monomórfico de estos loci. La designación de los electromorfos se realizó porcentualmente en forma relativa a la distancia desde el alelo más común. Con el propósito de

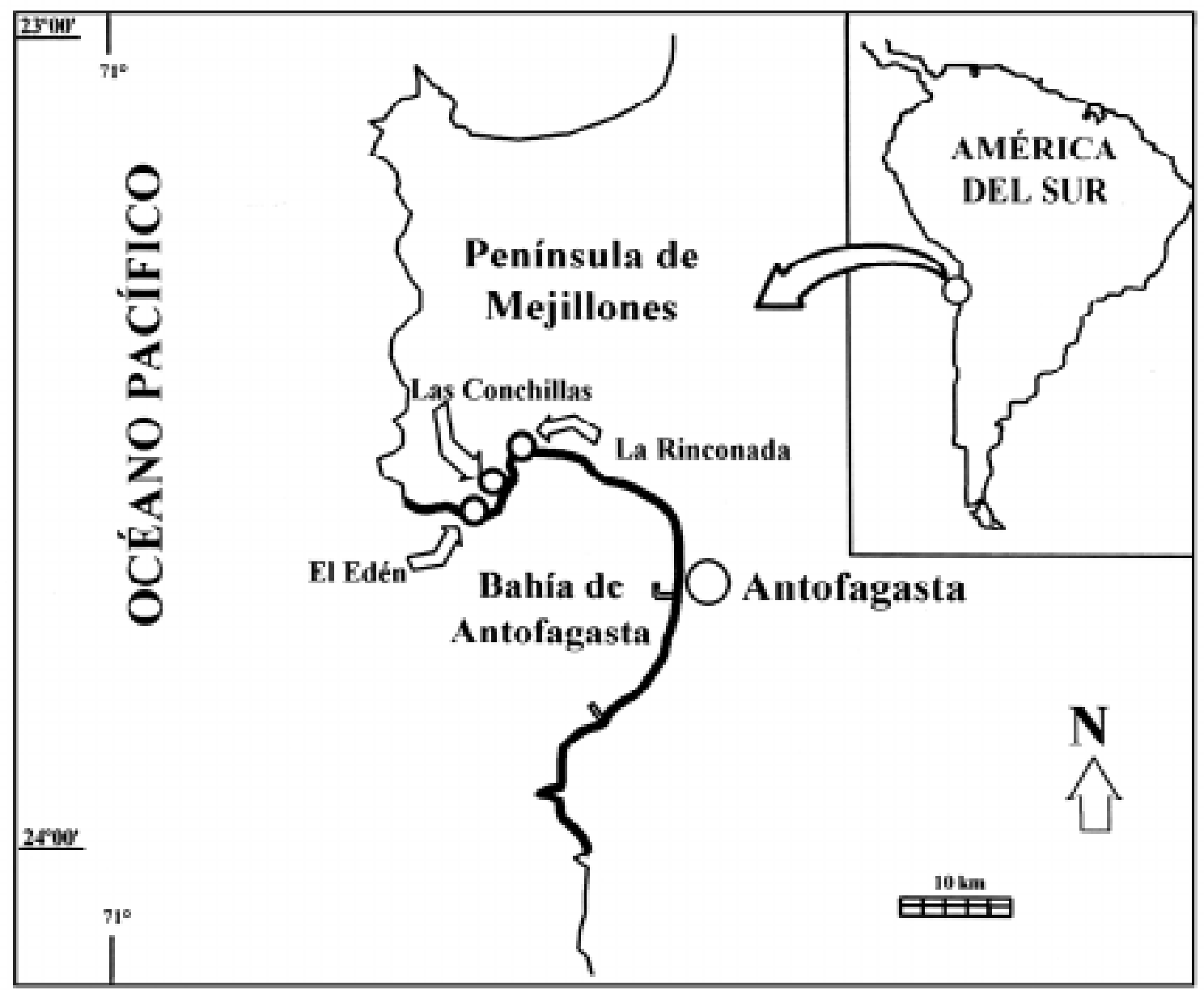

Fig. 1: Mapa de la Bahía de Antofagasta (Chile) donde se señalan las localidades de muestreo: El Edén, Las Conchillas y La Rinconada.

Map of Bay of Antofagasta (Chile), showing the sampling localities: El Edén, Las Conchillas and La Rinconada. 


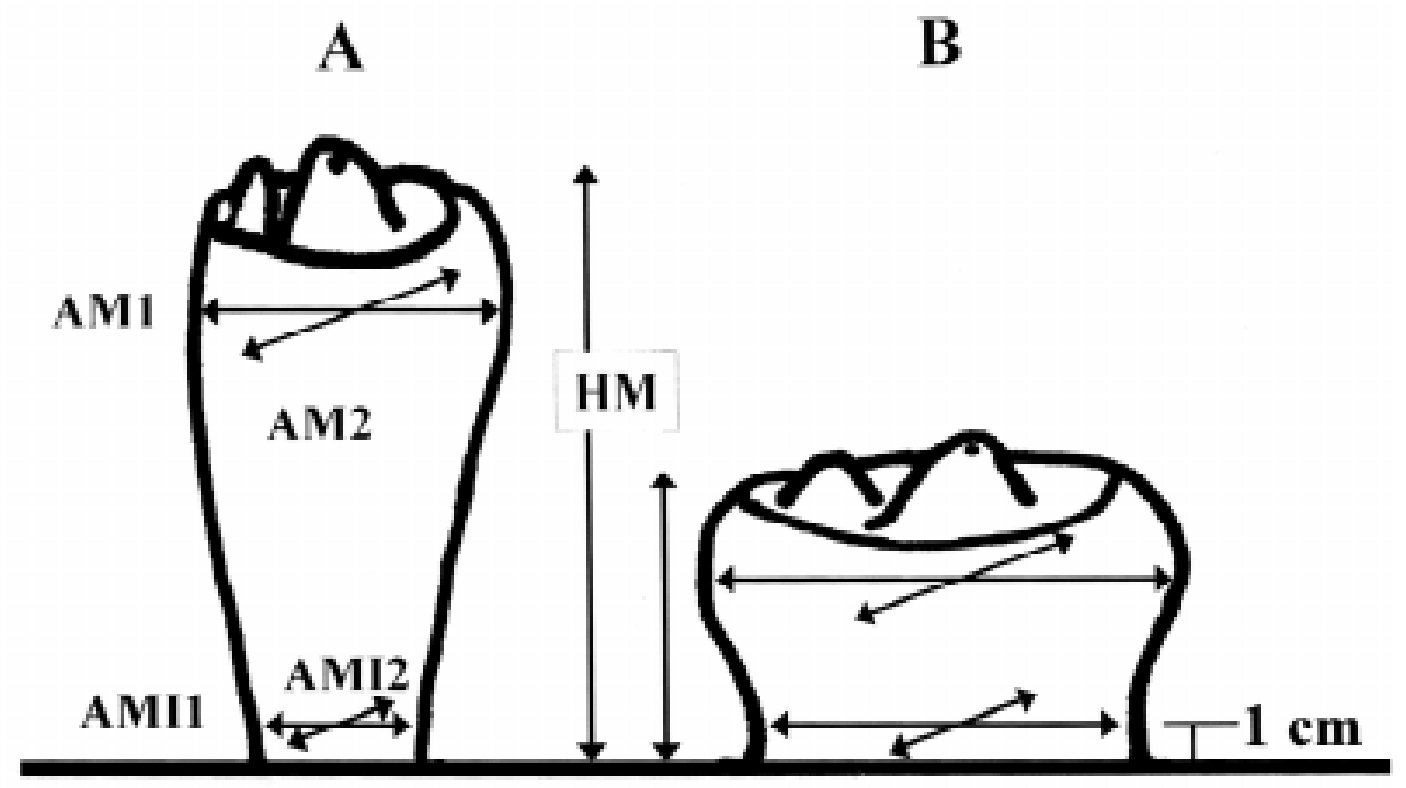

Fig. 2: Representación de los morfotipos (A) alargados y (B) achatados, y las medidas morfométricas analizadas. HM, Altura máxima; AM1, Ancho máximo superior 1 paralelo a los sifones; AM2, Ancho máximo superior 2 perpendicular a los sifones; AMI1, Ancho máximo inferior 1 paralelo a los sifones a $1 \mathrm{~cm}$ de la base y AMI2, Ancho máximo inferior 2 perpendicular a los sifones. El volumen (VOL) se estimó como:

$\mathrm{VOL}=[1 / 3 \cdot \mathrm{HM} \bullet(\{\pi \cdot \mathrm{AM} 1 / 2 \cdot \mathrm{AM} 2 / 2\}+\{\pi \cdot \mathrm{AMI} 1 / 2 \cdot \mathrm{AMI} 2 / 2\}+\sqrt{\pi \cdot \mathrm{AM} 1 / 2 \cdot \mathrm{AM} 2 / 2 \cdot \pi \bullet \mathrm{AMI} 1 / 2 \cdot \mathrm{AMI} 2 / 2})]$

Graphic representation of the (A) tall and (B) squat morphotypes, and the abbreviation of morphometric variables analyzed. HM, maximum height; AM1, maximum upper diameter 1 parallel to the siphons; AM2, maximum upper diameter 2 perpendicular to AM1; AMI, maximum lower diameter 1 parallel to the siphons at $1 \mathrm{~cm}$ from the base and AMI2, maximum lower diameter 1 perpendicular to AMI1. The formula used for the estimation of the volume (VOL) was the following:

$\mathrm{VOL}=[1 / 3 \bullet \mathrm{HM} \bullet(\{\pi \cdot \mathrm{AM} 1 / 2 \cdot \mathrm{AM} 2 / 2\}+\{\pi \cdot \mathrm{AMI} 1 / 2 \cdot \mathrm{AMI} 2 / 2\}+\sqrt{\pi \cdot \mathrm{AM} 1 / 2 \cdot \mathrm{AM} 2 / 2 \cdot \pi \cdot \mathrm{AMI} 1 / 2 \cdot \mathrm{AMI} 2 / 2})]$

determinar la magnitud de las diferencias genéticas entre los morfotipos y sobre la base de las frecuencias alélicas de los 17 loci analizados, se estimó los valores de identidad (I) y distancia genética insesgada(D) de Nei (1978), mediante el programa BIOSYS-1 (Swofford \& Selander 1981). Los estadísticos genéticos de frecuencias génicas, heterocigosidad, deficiencia de heterocigotos (d) y ajuste a Hardy-Weinberg (HW) se determinaron por morfotipo y localidad. El ajuste a las expectativas de Hardy-Weinberg (HW) se realizó mediante cadena de Markov (Raymond \& Rousset 1995a). Adicionalmente, se estimó los estadísticos F de Wright (Weir \& Cockerham 1984) y el número efectivo de migrantes bajo la presunción de un modelo neutro de deriva genética y migración (Waples 1987, Slatkin 1993). Los cálculos se realizaron con el programa GENEPOP 3.1. (Raymond \& Rousset 1995a). Para determinar la significancia estadística de la diferenciación genética poblacional entre morfotipos y localida- des, se aplicó la prueba exacta de Fisher (Raymond \& Roussett 1995b) y modelos log-normal usando PROC CATMOD de SAS (1996).

\section{RESULTADOS}

Se observó un mayor porcentaje $(55,9 \%)$ de individuos agregados para los morfotipos de piures achatados e intermedios $(51,4 \%)$ pero no así para los de morfotipo alargados $(45,9 \%)$. Sin embargo, estas diferencias no fueron significativas $(\mathrm{G}=$ $2,08 ;$ g.l. $=2 ; \mathrm{P}>0,25)$. La altura ajustada de los piures no fue significativamente afectada por el grado de agregamiento (solitario versus agregado) $(\mathrm{F}=0,42 ; \mathrm{P}=0,51)$. Se observó una distribución trimodal para las frecuencias de la altura ajustada, donde cada moda representa a cada uno de los tres morfotipos. La distribución porcentual de frecuencias de los morfotipos no fue significativamente distinta entre las localidades 
TABLA 1

Sistemas enzimáticos analizados, código de enzima (EC), tampones y número de loci polimórficos $(\mathrm{P})$ y monomórficos $(\mathrm{M})$

Enzymatic systems, enzyme commission (EC), buffer and polymorphic (P) and monomorphic (M) loci

\begin{tabular}{|c|c|c|c|c|c|}
\hline Enzima & Loci & Código & Tampón & $\mathrm{P}$ & $\mathrm{M}$ \\
\hline Anhidrasa carbónica & $\mathrm{CA}$ & 4.2.1.1 & 2 & 0 & 1 \\
\hline Aspartato aminotransferasa & AAT & 2.6.1.1 & 1 & 0 & 1 \\
\hline Enzima málica & EM & 1.1.1.38 & 2 & 0 & 1 \\
\hline Esterasa & EST & 3.1 .1 .1 & 1 & 0 & 2 \\
\hline Fosfoglucoisomerasa & PGI & 5.3 .1 .9 & 2 & 0 & 1 \\
\hline Fosfoglucomutasa & PGM & 5.4 .2 .2 & 2 & 1 & 0 \\
\hline Gliceraldehido-3-fosfato deshidrogenasa & GAPD & 1.2 .1 .12 & 1 & 0 & 2 \\
\hline Glioxalasa & GLIO & 4.4.1.5 & 1 & 0 & 1 \\
\hline Glicerol-3-fosfato deshidrogenasa & GPD & 1.1 .1 .8 & 2 & 0 & 1 \\
\hline Isocitrato deshigrogenasa & IDH & 1.1.1.42 & 2 & 1 & 1 \\
\hline Malato deshidrogenasa & $\mathrm{MDH}$ & 1.1 .1 .37 & 2 & 1 & 0 \\
\hline Proteínas totales & PT & —_- _- & 1 & 0 & 2 \\
\hline \multirow[t]{2}{*}{ Tetrazolio oxidasa } & TO & 1.12 .1 .1 & 2 & 0 & 1 \\
\hline & & Total & & 3 & 14 \\
\hline
\end{tabular}

Tampón: (1) Poulik pH 8,2-8,7; (2) Tris Cítrico pH 7,0

de El Edén y Las Conchillas (promedio $=33,3 \%$; $\mathrm{G}=0,30 ;$ g.l. $=2 ; \mathrm{P}>0,86)$, pero estas dos localidades fueron significativamente distintas de La Rinconada $(\mathrm{G}=11,55$; g.l. $=2, \mathrm{P}<0,005)$, donde se observó el menor porcentaje de morfotipos achatados $(10,5 \%)$ y el mayor porcentaje de alargados $(56,6 \%)$.

En el intermareal, las velocidades promedios del oleaje difieren significativamente entre las tres localidades: El Edén $\left(6,6 \mathrm{~m} \mathrm{~s}^{-1}\right)$, Las Conchillas $\left(5,7 \mathrm{~m} \mathrm{~s}^{-1}\right)$ a La Rinconada $\left(3,8 \mathrm{~m} \mathrm{~s}^{-1}\right)$ $(\mathrm{F}=21,47 ; \mathrm{P}<0,001)$. Se observó un gradiente de la velocidad máxima del oleaje desde El Edén (10 $\left.\mathrm{m} \mathrm{s}^{-1}\right)$, Las Conchillas $\left(8,8 \mathrm{~m} \mathrm{~s}^{-1}\right)$ a La Rinconada $\left(4,7 \mathrm{~m} \mathrm{~s}^{-1}\right)$. La diferencia entre El Edén y Las Conchillas fue marginalmente significativa (Tukey-HSD; $\mathrm{P}=0,054$ ), pero ambas localidades difieren significativamente de La Rinconada (Tukey-HSD; P < 0,002), la localidad de menor exposición al oleaje y con las menores velocidades promedios del oleaje.

Para los 17 loci analizados se determinó un total de 29 alelos, de los cuales el $100 \%$ fue compartido entre los morfotipos. El polimorfismo medio fue idéntico entre los tres morfotipos $(\mathrm{P}=$ $17,6 \%)$, y la heterocigosidad media no fue significativamente distinta entre ellos: achatados $(\mathrm{H}=0,051)$, intermedios $(\mathrm{H}=0,055)$ y alargados $(\mathrm{H}=0,051)$ (prueba $\mathrm{U}$ de Mann-Whitney; $\mathrm{P}>$ $0,93)$. La identidad genética promedio entre morfotipos fue de $\mathrm{I}=0,995(\mathrm{DE}=0,001)$ y la distancia genética de $\mathrm{D}=0,005(\mathrm{DE}=0,001)$. Las frecuencias alélicas de los loci polimórficos para la interacción entre localidad y morfotipo no fueron significativamente distintas (modelo log-normal, Chi-cuadrado $=3,23 ;$ g.l. $=4, \mathrm{P}>0,52)$, lo que permitió realizar los análisis de las frecuencias génicas separadamente entre localidades y entre morfotipos (Tabla 2). Sólo el locus PGM-1 mostró una deficiencia de heterocigotos (d negativos) y un alejamiento significativo respecto de las expectativas de Hardy-Weinberg, para cada localidad $(\mathrm{P}<0,027)$ y morfotipo $(\mathrm{P}<0,029)$ (Tabla 2). Entre los morfotipos no hubo diferencias significativas de las frecuencias alélicas para los tres loci polimórficos ( $\mathrm{P}>0,273$; Tabla 2$)$ y al mismo tiempo los valores de $\mathrm{F}_{\mathrm{st}}$ no fueron significativamente distintos de cero (Tabla 2). Sin embargo, entre las localidades las frecuencias alélicas fueron significativamente distintas para los loci IDH-1 y PGM-1 (prueba de Fisher, P < 0,036; Tabla 2), con valores de $\mathrm{F}_{\text {st }}$ significativamente mayores a cero. La comparación a posteriori de las frecuencias génicas entre pares de localidades, mostró que para el locus PGM-1 todas las comparaciones fueron significativamente distintas (prueba de Fisher; $\mathrm{P}<0,013$ ), observándose una clina estadísticamente significativa en las frecuencias de tres alelos del locus PGM-1 (G $=14,25 ; \mathrm{gl}=4 ; \mathrm{P}<0,025)$ entre El Edén y La Rinconada, con un decremento de la heterocigosidad y un incremento en la deficiencia de heterocigotos (Tabla 2). En tanto, para el locus IDH-1 las diferencias de las frecuencias alélicas fueron significativas sólo para la comparación entre las localidades extremas de El Edén y La 
Rinconada (Fisher; $\mathrm{P}<0,02$ ). Estas diferencias están asociadas con el incremento de la frecuencia de uno de los alelos raros (97), el incremento de la heterocigosidad y un decremento de la deficiencia de heterocigotos (Tabla 2). El número de migrantes $\left(\mathrm{N}_{\mathrm{m}}\right)$ estimado entre localidades fue de 10,6 y entre morfotipos de 23,5 individuos/generación.
Las tres variables cuantitativas: volumen y pesos húmedos de la túnica y del cuerpo, fueron afectadas significativamente por los efectos de localidad, de morfotipo y de su interacción (ANMUDEVA; P < 0,045). El primer eje del análisis canónico explica significativamente $(\mathrm{P}=$ $0,015)$ un $12 \%$ de la varianza y los coeficientes canónicos estandarizados del primer eje muestran

TABLA 2

Frecuencias alélicas y estadísticos genéticos para cada locus polimórfico, morfotipo y localidad. Se incluye el número de genes analizados $(2 \mathrm{~N})$, heterocigosidad observada $(\mathrm{H})$, deficiencia de heterocigotos (d) y probabilidad para ajuste a Hardy-Weinberg -P(HW). Fst es el estadístico de Wright y $\mathrm{P}$ es la probabilidad de la significación de la diferencia génica entre morfotipos y localidades

Allelic frequency for the polymorphic loci, number of analyzed genes $(2 \mathrm{~N})$, observed heterozigosity $(\mathrm{H})$, heterozygote deficiency $(\mathrm{d})$ and probability for fit to Hardy-Weinberg - $\mathrm{P}(\mathrm{HW})$ by morphotypes and localities. With genic differentiation probability $(\mathrm{P})$ and $\mathrm{F}_{\mathrm{st}}$ index between morphotypes and localities

\begin{tabular}{|c|c|c|c|c|c|c|c|}
\hline \multirow[t]{2}{*}{ Locus } & \multirow[b]{2}{*}{ Alelo } & \multicolumn{3}{|c|}{ Localidad } & \multicolumn{3}{|c|}{ Morfotipo } \\
\hline & & El Edén & Las Conchillas & La Rinconada & Achatado & Intermedio & Alargado \\
\hline \multirow[t]{3}{*}{ MDH-1 105} & 0,022 & 0,009 & 0,018 & 0,022 & 0,008 & 0,020 & \\
\hline & 100 & 0,963 & 0,947 & 0,964 & 0,944 & 0,958 & 0,969 \\
\hline & 95 & 0,015 & 0,044 & 0,018 & 0,033 & 0,034 & 0,010 \\
\hline $2 \mathrm{~N}$ & & 136 & 114 & 56 & 90 & 118 & 98 \\
\hline $\mathrm{H}$ & & 0,074 & 0,105 & 0,071 & 0,111 & 0,085 & 0,061 \\
\hline $\mathrm{d}$ & & 0,021 & 0,039 & 0,009 & 0,032 & 0,028 & 0,014 \\
\hline $\mathrm{P}(\mathrm{HW})$ & & 1,000 & 1,000 & 1,000 & 1,000 & 1,000 & 1,000 \\
\hline $\mathrm{P}$ & & 0,596 & & & 0,691 & & \\
\hline $\mathrm{F}_{\mathrm{st}}$ & & 0,002 & & & 0,006 & & \\
\hline \multirow[t]{9}{*}{ PGM-1 } & 115 & 0,000 & 0,014 & 0,069 & 0,000 & 0,033 & 0,038 \\
\hline & 110 & 0,103 & 0,121 & 0,042 & 0,138 & 0,067 & 0,100 \\
\hline & 103 & 0,103 & 0,100 & 0,125 & 0,087 & 0,142 & 0,075 \\
\hline & 100 & 0,132 & 0,329 & 0,292 & 0,287 & 0,250 & 0,287 \\
\hline & 97 & 0,147 & 0,043 & 0,042 & 0,075 & 0,075 & 0,050 \\
\hline & 95 & 0,147 & 0,086 & 0,181 & 0,150 & 0,083 & 0,162 \\
\hline & 90 & 0,132 & 0,164 & 0,069 & 0,112 & 0,167 & 0,100 \\
\hline & 80 & 0,162 & 0,093 & 0,167 & 0,087 & 0,125 & 0,175 \\
\hline & 75 & 0,074 & 0,050 & 0,014 & 0,063 & 0,058 & 0,013 \\
\hline $2 \mathrm{~N}$ & & 68 & 140 & 72 & 80 & 120 & 80 \\
\hline $\mathrm{H}$ & & 0,735 & 0,671 & 0,611 & 0,600 & 0,683 & 0,725 \\
\hline d & & $-0,17$ & $-0,19$ & $-0,27$ & $-0,29$ & $-0,21$ & $-0,14$ \\
\hline $\mathrm{P}(\mathrm{HW})$ & & 0,027 & 0,001 & 0,001 & 0,001 & 0,029 & 0,003 \\
\hline $\mathrm{P}$ & & 0,001 & & & 0,301 & & \\
\hline $\mathrm{F}_{\mathrm{st}}$ & & 0,023 & & & 0,006 & & \\
\hline \multirow[t]{3}{*}{ IDH-1 } & 110 & 0,056 & 0,020 & 0,043 & 0,038 & 0,066 & 0,000 \\
\hline & 100 & 0,935 & 0,941 & 0,848 & 0,925 & 0,896 & 0,970 \\
\hline & 97 & 0,009 & 0,039 & 0,109 & 0,038 & 0,038 & 0,030 \\
\hline $2 \mathrm{~N}$ & & 108 & 102 & 46 & 80 & 106 & 66 \\
\hline $\mathrm{H}$ & & 0,093 & 0,118 & 0,304 & 0,150 & 0,169 & 0,086 \\
\hline d & & $-0,25$ & 0,038 & 0,113 & 0,047 & $-0,12$ & 0,030 \\
\hline $\mathrm{P}(\mathrm{HW})$ & & 0,187 & 1,000 & 1,000 & 1,000 & 0,256 & 1,000 \\
\hline $\mathrm{P}$ & & 0,036 & & & 0,273 & & \\
\hline $\mathrm{F}_{\mathrm{st}}$ & & 0,033 & & & 0,012 & & \\
\hline
\end{tabular}


que es el peso de la túnica el que tiene la mayor influencia en la interacción entre localidad y morfotipo. El ANDEVA univariado mostró que los efectos de localidad y de la interacción localidad x morfotipo fueron significativos para el peso húmedo de la túnica $(\mathrm{P}<0,048)$; en tanto que el efecto morfotipo fue significativo sólo para los pesos húmedos de la túnica y del cuerpo ( $\mathrm{P}<$ $0,001)$, pero no para el volumen $(P=0,209)$. Los tres morfotipos difieren significativamente entre sí en el peso húmedo del cuerpo (Tukey-HSD; P < 0,001), siendo el morfotipo achatado de menor peso y los alargados de mayor peso.

Debido a que el efecto de la interacción (localidad x morfotipo) fue significativo para el peso húmedo de la túnica, se comparó las diferencias para cada morfotipo entre las tres localidades usando el procedimiento de "slice" de PROC GLM (SAS 1996). Sólo el morfotipo alargado mostró diferencias significativas entre las tres localidades $(\mathrm{F}=3,97 ; \mathrm{P}=0,021)$, siendo significativamente menos pesado en la localidad de El Edén (Tukey-HSD; P < 0,001) en relación con las otras dos localidades.

\section{DISCUSIÓN}

Los valores de identidad y distancia genética sugieren que las diferencias genéticas entre los morfotipos de $P$. praeputialis de Antofagasta son muy bajas y están representadas por valores de I $=0,995 \mathrm{y} \mathrm{D}=0,005$. Esto es concordante con el hecho de que los morfotipos comparten el $100 \%$ de los 29 alelos determinados y presentan valores significativamente similares de polimorfismo y heterocigosidad medios. Estos resultados son muy distintos de los obtenidos para los morfotipos australianos, en los que se ha estimado una identidad genética de $\mathrm{I}=0,69$ y una distancia genética de $\mathrm{D}=0,37$ (Killingly 1976). Además en seis loci (LAP-2, MDH, 6PGD, PGI, PT-3 y TO) de los 16 analizados en los morfotipos australianos se encontró $22(52 \%)$ alelos diagnósticos no compartidos, de un total de 42 alelos determinados (Killingly 1976). Cuatro de estos loci diagnósticos fueron resueltos para Antofagasta, pero no se encontró ningún alelo diagnóstico (como aquellos reportados por Killingly 1976) que diferenciara a los tres morfotipos. El hecho de que sólo se estudió 10 individuos para los loci monomórficos no es un sesgo, en tanto bastaría sólo un individuo para determinar la presencia de un alelo diagnóstico.

De acuerdo a Thorpe (1982), en el $98 \%$ de los casos valores de identidad genética (I) sobre 0,85 son esperados para diferencias intraespecíficas y bajo ese valor se esperan diferencias interespecíficas. En consecuencia, los valores observados promedios entre los morfotipos de Antofagasta son mucho mayores que el valor crítico de $\mathrm{I}=0,85$, lo que sugiere que la hipótesis de que estos morfotipos sean especies diferentes debiera ser descartada. En cambio, los valores de I obtenidos para los morfotipos australianos, menores que el valor crítico, sugiere el estatus de dos especies distintas. Sin embargo, no ha existido un esfuerzo sistemático para nominar y definir los caracteres diagnósticos de la presunta nueva especie representada por el morfotipo achatado amarillo. La coloración amarilla no sólo de la túnica sino de los órganos internos (Dalby 1997a, 1997b), que nunca se ha observado en $P$. praeputialis de Antofagasta (J.C. Castilla resultados no publicados), sería uno de los rasgos distintivos de la presunta nueva especie representada por el morfotipo achatado australiano. Los morfotipos café estudiados en Antofagasta debieran ser variantes morfológicas del morfotipo alargado de Australia y de sus variantes intermedias de coloración café (Kott 1952, 1976) de la misma especie, $P$. praeputialis. De hecho, el polimorfismo y la heterocigosidad medios (excluyendo el locus PGM altamente variable y no analizado por Killingly 1976) obtenido para los morfotipos de Antofagasta es similar con el informado por Killingly (1976) para el morfotipo alargado ( $=P$. praeputialis) de Australia (Antofagasta $\mathrm{H}=0,015$; Australia $\mathrm{H}=0,014 ; \mathrm{U}=159,0 ; \mathrm{P}>$ $0,255)$. También, el comportamiento electroforético de algunos loci sugiere esta misma relación entre los morfotipos, puesto que tanto el morfotipo australiano alargado (Killingly 1976) como los tres morfotipos de Antofagasta, son monomórficos para el locus PGI y polimórficos para los loci IDH y MDH. Con la excepción de la MDH que fue monomórfica en los sitios trabajados por Dalby (1997a) en Australia. Esta discrepancia podría explicarse por los pequeños tamaños muestreales usados por Dalby (1997a) ( $<11$ por localidad), por la presunta diferenciación genética geográfica del morfotipo alargado en Australia, o en el caso de Antofagasta podría deberse además a cambios ocurridos en el proceso de colonización de $P$. praeputialis en Chile, o a que las localidades de origen de los colonizadores fueran distintas a las estudiadas por Dalby (1997a). El piure de Antofagasta habría colonizado la Bahía recientemente presumiblemente transportados por barco como fauna incrustante o en agua de lastre, o alternativamente transportados mediante su adhesión a macroalgas u objetos flotantes arrastrados por las corrientes oceánicas (Castilla \& Guiñez 2000, Castilla et al. en prensa a). En el primer reporte científico de su presencia en la Bahía de 
Antofagasta fue confundido con la especie Pyura chilensis (Guiler 1959). Sin embargo, personas ancianas de Antofagasta indican que poblaciones intermareales de $P$. praeputialis estaban ya presente entre 1920 y 1925 (Castilla et al. en prensa a). Los resultados obtenidos en este trabajo, sugieren que él o los eventos de colonización en Antofagasta, sólo habrían sido posible para individuos de la especie $P$. praeputialis y que habrían eventualmente incluido individuos del morfotipo alargado, y no para los individuos de la supuesta nueva especie australiana representada por el morfotipo achatado amarillo. La posible determinación, mediante técnicas moleculares de ADN, de él o los lugares de origen en Australia de los colonizadores en Chile permitirá determinar los cambios en la estructura genética poblacional aloenzimática y molecular asignables al proceso de colonización en Antofagasta.

La evidencia discutida arriba sugiere entonces que las diferencias morfológicas en el peso y la forma de los morfotipos de Antofagasta, no obedece a diferencias genéticas interespecíficas como es el caso de los morfotipos australianos, sino que por el contrario, debiera tener una base intraespecífica. Sin embargo, ella no estaría asociada con la variación genética observada en los tres loci polimórficos estudiados, puesto que no se observó diferencias significativas en las frecuencias alélicas entre los morfotipos. Por lo tanto, las diferencias podrían ser explicadas como respuestas plásticas moduladas ambientalmente o determinadas por procesos selectivos y genéticos asociados con otros loci polimórficos o sistemas poligénicos. En organismos marinos existen antecedentes de caracteres que responden plásticamente a factores ambientales. Así, en corales existen respuestas plásticas de la morfología del esqueleto y de la colonia moduladas por la intensidad de la exposición solar o la profundidad del arrecife, respectivamente (Foster 1979, 1980, West et al. 1993). En el cirripedio Chthamalus anisopoma la forma es modulada por la depredación (Lively 1986). En moluscos, la forma de la concha es una respuesta plástica al estrés térmico y la intensidad del oleaje (De Wolf et al. 1997, Leonard et al. 1999) y en crustáceos, las variaciones en los apéndices y el tamaño del cuerpo son moduladas por diferentes condiciones oceanográficas (Sarda et al. 1998). En hidróides coloniales (Yund 1991) y en el piure de Antofagasta (Castilla et al. 2000, Guiñez \& Castilla 2001) la evidencia sugiere que la forma sería modulada por la competencia intraespecífica asociada con el grado de agregamiento. Sin embargo, respecto de este último factor, nuestros resultados muestran que la forma de los morfotipos no sería modulada por el agregamiento, puesto que éste no afecta signifi- cativamente a la altura ajustada, el índice de forma utilizado.

En el género Pyura se ha propuesto que la túnica es un rasgo adaptativo que representa un mecanismo avanzado de protección en contra de la depredación y la acción del oleaje (Day 1974), por lo que sería esperable un componente genético de varianza aditiva para el rasgo peso de la túnica, si este es adaptativo. Al respecto, es sugerente el hecho de que en localidades más expuestas (El Edén y Las Conchillas) los individuos más alargados sean menos frecuentes y que presenten también un menor peso de la túnica, en relación al sitio más protegido (La Rinconada). Así, es posible que estas diferencias sean, al menos en parte, una respuesta a la intensidad del oleaje, o a otros factores como la depredación, que puedan covariar con el gradiente. En organismos marinos, en particular intermareales, se conoce de repuestas a factores mecánicos asociados con la intensidad del oleaje. Por ejemplo, en gasterópodos existen presiones selectivas diferenciales determinadas por el gradiente de intensidad del oleaje, que explican el hecho de que en lugares más protegidos sean más frecuentes morfotipos más alargados, esculpidos y de conchas gruesas y pesada, y por el contrario, en sitios expuestos a mayores intensidades del oleaje los morfotipos sean de tamaño pequeño y sus conchas más livianas y lisas (Vermeij 1978, Reimchen 1981, Denny et al. 1985, Sundberg 1988, Boulding \& Van Alstyne 1993, Frid \& Fordham 1994, Chapman 1995, Preston et al. 1996, Denny 1998, De Wolf et al. 1997). Una situación similar podría ocurrir en el gradiente de intensidad del oleaje con la forma y los pesos de la túnica y del cuerpo en los morfotipos de piures de Antofagasta. Sin embargo, esto no explicaría el hecho de que en Australia el morfotipo alargado de piure es más frecuente en costas abiertas con mayor exposición al oleaje (Dalby 1997a, 1997b). Por otra parte, no se puede descartar que la depredación pudiera interactuar con la intensidad del oleaje y explicar las diferencias discutidas, puesto que, de los depredadores del piure en Antofagasta (Paine \& Suchanek 1983, Pacheco \& Castilla 2000) (gasterópodos: Thais stramonita chocolata, Thais haemastoma, Nassarius gayi y Priene rude; resultados no publicados; asteroideos: Heliaster helianthus y Stichaster striatus y aves: Haematopus palliatus pitanay y Haematopus ater), se conoce que las aves consumen individuos pequeños de piures y preferentemente en sitios de baja exposición al oleaje (Pacheco \& Castilla 2000).

Las diferencias genéticas observadas no están asociadas con los morfotipos como se discutió arriba, sino con las diferencias significativas de las frecuencias alélicas de dos loci IDH-1 y PGM-1 
entre localidades, con una clina de los alelos raros y de la heterocigosidad entre El Edén y La Rinconada. En tunicados marinos se tiene casos en que la diferenciación geográfica se explica por deriva genética y restricciones del flujo génico (e.g., Cohen 1990, Grosberg 1991, Yund 1995). Sin embargo, de acuerdo a la teoría neutral (Allendorf 1983, Slatkin 1985, Slatkin \& Barton 1989) para valores del número de migrantes $\mathrm{N}_{\mathrm{m}}$ mayores a 1 , y en particular tan altos como los estimados en este trabajo $\left(\mathrm{N}_{\mathrm{m}}=10,6\right)$, se espera la homogeneidad de las frecuencias génicas entre poblaciones, si sólo la deriva genética y la migración son los factores que determinan la estructura genética geográfica. En nuestro caso el efecto de la deriva genética es poco probable que explique las diferencias observadas, debido a la alta densidad de individuos por unida de área estimados para el piure en Antofagasta (más de 1.600 ind m$^{-2}$, Castilla et al. 2000). Por otra parte, se espera una alta tasa migracional entre las tres localidades estudiadas $(10 \mathrm{~km}$ de distancia entre las localidades extremas, Fig. 1), probablemente determinada por celdas de circulación costera con un vector predominante desde El Edén hacia La Rinconada (Escribano \& Hidalgo 2001). Sin embargo, en $P$. praeputialis es esperable también la existencia de restricciones al flujo génico debido a la corta vida planctónica de su larva (Clarke et al. 1999) y al probable efecto de retención de las larvas, a escala de localidad, que pueden generar procesos oceanográficos costeros muy activos al interior de la Bahía de Antofagasta, tales como, las zonas de convergencia de alisamientos ("slicks") costeros, las resacas de mareas internas ("internal tidal bores") y líneas de circulación de Langmuir (ver Castilla et al. en prensa b). A este respecto se ha sugerido la hipótesis de que procesos de mesoescala $(\mathrm{km})$ como mecanismos de circulación del mar, tales como sombras y frentes de surgencia al interior de la Bahía pueden también facilitar la retención de la larva dentro de la Bahía de Antofagasta y explicar su rango de distribución tan restringido en Chile (Clarke et al. 1999, Castilla et al. en prensa b). Alternativamente, pudieran existir presiones selectivas actuando en el gradiente de intensidad del oleaje, que por una parte limitan el uso de estimadores como el número de migrantes y por otra podrían explicar las diferencias poblacionales observadas. Son escasos los trabajos que muestran procesos selectivos que actúan sobre la variabilidad aloenzimática modulados por la intensidad del oleaje en gradientes de flujo génico; por ejemplo, para el gasterópodo Nucella lapillus, esto explicaría que en los sitios más protegidos del oleaje existe un incremento de la variabilidad genética y del polimorfismo enzimático en relación con los sitios más expuestos (Goudet et al.
1994). En nuestro trabajo el locus IDH-1 muestra este mismo patrón, pero el locus PMG-1 muestra el patrón inverso. Lo que podría sugerir la existencia de presiones selectivas diferenciales que actúan sobre estos loci o loci íntimamente ligados a ellos en el gradiente de intensidad del oleaje.

La estructura genética geográfica observada y la variabilidad de los rasgos estudiados en los morfotipos de $P$. praeputialis, tanto como su forma, serían la consecuencia del tipo de evento y de la historia de la colonización de la Bahía de Antofagasta, tanto como de la acción de factores ambientales neutros o selectivos en el presente. Para avanzar en el conocimiento de estos aspectos y al mismo tiempo para poner a prueba las hipótesis sugeridas en este trabajo se hace necesario, incrementar el número de localidades a estudiar en la Bahía, realizar experimentos de traslados recíprocos de individuos de piures entre localidades dentro y fuera del gradiente de intensidad del oleaje, incrementar el número de loci enzimáticos o moleculares y estimar la significancia de la varianza genética aditiva y de las correlaciones fenotípicas, genéticas y ambientales (Falconer \& Mackay 1996) de los rasgos que varían entre los morfotipos (i.e. peso de la túnica y del cuerpo) con la adecuación darwiniana.

\section{AGRADECIMIENTOS}

Se agradece a Marcela Clarke por la colaboración en el procesamiento de las muestras. A Raquel Pinto, Jorge Alvarado, Cristián Pacheco y Mauricio Cerda por el apoyo en terreno. A Elie Poulin y dos árbitros anónimos por sus sugerencias críticas. El trabajo fue financiado por los proyectos Cátedra Presidencial en Ciencia (1997) al Dr. Juan Carlos Castilla, Convenio Minera Escondida Ltda-Pontificia Universidad Católica de Chile y Proyecto Fundación Mellon-P. U. Católica de Chile (J.C. Castilla \& S. Navarrete). Este trabajo es parte de la Tesis Doctoral en Ciencias Biológicas-área Zoología (Universidad de Concepción) del primer autor. El trabajo se completó bajo el auspicio del proyecto FONDAPFONDECYT 1501-0001 al Centro de Estudios Avanzados en Ecología \& Biodiversidad.

\section{LITERATURA CITADA}

ACHITUV Y \& L MIZRAHI (1987) Allozyme differences between tidal levels in Tetraclita squamosa Pilsbry from the Red Sea. Journal of Experimental Marine Biology and Ecology 108: 181-189. 
ALLENDORF FW (1983) Isolation, gene flow, and genetic differentiation among populations. En: SchonewaldCox CM, SM Chambers, B MacBryde \& L Thomas (eds) Genetics and conservation: 51-65. Benjamin/ Cummings, London, United Kingdom.

BERTNESS MD, SD GAINES \& SM YEH (1998) Making mountains out of barnacles: the dynamics of acorn barnacle hummocking. Ecology 79: 1382-1394.

BOULDIN EG \& KL VAN ALSTYNE (1993) Mechanisms of differential survival and growth of two species of Littorina on wave-exposed and on protected shores. Journal of Experimental Marine Biology and Ecology 108: 181-189.

CASTILLA JC (1981) Perspectivas de investigación en estructura y dinámica de comunidades intermareales rocosas de Chile central. II. Depredadores de alto nivel trófico. Medio Ambiente (Chile) 5: 190-215.

CASTILLA JC (1998) Las comunidades intermareales de la Bahía de Antofagasta: estudios de línea base y el programa ambiental de Minera Escondida Limitada en Punta Coloso. En: Arcos D (ed) Minería del cobre, ecología y ambiente costero: el caso de Minera Escondida Ltda.: 191-214. Editorial Aníbal Pinto, Concepción, Chile.

CASTILLA JC \& R GUIÑEZ (2000) Disjoint geographical distribution of intertidal and nearshore benthic invertebrates in the Southern Hemisphere. Revista Chilena de Historia Natural 73: 585-603.

CASTILLA JC, DK STEINMILLER \& CJ PACHECO (1998) Quantifying wave exposure daily and hourly on the intertidal rocky shore of central Chile. Revista Chilena de Historia Natural 71: 19-25.

CASTILLA JC, R GUIÑEZ, J ALVARADO, C PACHECO \& M VARAS (2000) Distribution, population structure, population biomass and morphological characteristics of the tunicate Pyura stolonifera in the Bay of Antofagasta, Chile. Marine Ecology 21: 114.

CASTILLA JC, AG COLLINS, CP MEYER, R GUIÑEZ \& DR LINDBERG (en prensa a) Recent introduction of the dominant tunicate, Pyura praeputialis (Urochordata, Pyuridae) to Antofagasta, Chile. Molecular Ecology.

CASTILLA JC, N LAGOS, R GUIÑEZ \& J LARGIER (en prensa b) Embayments and nearshore retention of plankton: the Antofogasta Bay, Chile and other examples. En: Castilla JC \& J Largier (eds) Connections and dynamics of coastal litoral systems: open coast and embayments. Ediciones Universidad Católica de Chile, Santiago, Chile.

CLARKE M, V ORTIZ \& JC CASTILLA (1999) Does early development of the Chilean tunicate Pyura praeputialis (Heller, 1878) explain the restricted distribution of the species? Bulletin of Marine Science 65: 745-754.

COHEN S (1990) Outcrossing in field populations of two species of self-fertile ascidians. Journal of Experimental Marine Biology and Ecology 140: 147-158.

CHAPMAN MG (1995) Spatial patterns of shell shape of three species of co-existing littorinid snails in New South Wales, Australia. Journal of Molluscan Studies 61: 141-162.
DALBY JE JR (1997a) Reproductive and electrophoretic evidence for genetic maintenance of dimorphism in the ascidian Pyura stolonifera near Melbourne, Australia. Ophelia 47: 227-243.

DALBY JE Jr (1997b) Dimorphism in the ascidian Pyura stolonifera near Melbourne, Australia, and its Evaluation through field transplant experiments. Marine Ecology 18: 253-271.

DANDO PR \& AJ SOUTHWARD (1981) Existence of 'Atlantic' and 'Mediterranean' forms of Chthamalus montagui (Crustacea: Cirripedia) in the western Mediterranean. Marine Biology Letters 2: 239-248.

DAY RW (1974) An investigation of Pyura stolonifera (Tunicata) from the Cape Town Peninsula. Zoologica Africana 9: 35-58.

DEGNAN BM \& MF LAVIN (1995) Highly repetitive DNA sequences provide evidence for a lack of gene flow between two morphological forms of Herdmania momus (Ascidiacea: Stolidobranchia). Marine Biology 124: 293-299.

DENNY MW (1998) Biology and mechanisms of the wave swept environment. Princeton University Press, Princeton, New Jersey. 329 pp.

DENNY MW, TL DANIEL \& MAR KOEHL (1985) Mechanical limits to size in wave-swept organisms. Ecological Monographs 55: 69-102.

DE WOLF $\mathrm{H}, \mathrm{T}$ BACKELJAU, R MEDEIROS \& $\mathrm{R}$ VERHAGEN (1997) Microgeographical shell variation in Littorina striata, a planktonic developing periwinkle. Marine Biology 129: 331-342.

ESCRIBANO R \& P HIDALGO (2001) Circulación inducida por el viento en Bahía de Antofagasta norte de Chile $\left(23^{\circ} \mathrm{S}\right)$. Revista de Biología Marina y Oceanografía (Chile) 36: 43-60.

FALCONER DS \& TFC MACKAY (1996) Introduction to quantitative genetics, Fourth edition. Longman Group Ltd., Harlow, United Kingdom. 464 pp.

FLOWERDEW MW \& DJ CRISP (1975) Esterase heterogeneity and an investigation into racial differences in the cirrripede Balanus balanoides using acrylamide gel electrophoresis. Marine Biology 33: 33-39.

FOSTER AB (1979) Phenotypic plasticity in the reef corals Montastraea annularis (Ellis \& Solander) and Siderastrea siderea (Ellis \& Solander). Journal Experimental Marine Biology and Ecology 39: 25-54.

FOSTER AB (1980) Environmental variation in skeletal morphology within the Caribbean reef corals Montastraea annularis and Siderastrea siderea. Bulletin of Marine Science 30: 678-709.

FRID JLC \& E FORHAM (1994) The morphology of the sublitoral gastropod Gibbula cineraria (L.) along a gradient of wave action. Ophelia 40: 135-146.

GOUDET J, T DE MEEÚS, AJ DAY \& CJ GLIDDON (1994) The different levels of population structuring of the dogwhelk, Nucella lapillus, along the south devon coast. En: Beaumont AR (ed) Genetics and evolution of aquatic organisms: 81-95. Chapman \& Hall, London, United Kingdom.

GROSBERG RK (1991) Sperm-mediated gene flow and the genetic structure of a population of the colonial ascidian Botryllus schlosseri. Evolution 45: 130-142. 
GUILER ER (1959) Intertidal belt-forming species on the rocky coasts of northern Chile. Papers and Proceedings of the Royal Society of Tasmania 93: 33-58.

GUIÑEZ R \& JC CASTILLA (2001) An allometric tridimensional model of self-thinning for a gregarious tunicate. Ecology 82: 2331-2341.

HARRIS H \& DA HOPKINSON (1976) Handbook of enzyme electrophoresis in human genetics, supplement, North-Holland Publishing Company, Amsterdam, The Netherlands. 115 pp.

JOHANESSON B \& K JOHANESSON (1990) Littorina neglecta Bean, a morphological form within the variable species Littorina saxatilis (Olivi)? Hydrobiologia 193: 71-87.

JOHANESSON K, B JOHANESSON \& E ROLANALVAREZ (1993) Morphological differentiation and genetic cohesiveness over a microenvironmental gradient in the marine snail Littorina saxatilis. Evolution 47: 1770-1787.

KILLINGLY DJ (1976) Biochemical population genetics of cunjevoi. Bachelor of Science (Hons.) Thesis, University of Sydney, Sydney, Australia. $70 \mathrm{pp}$.

KOTT P (1952) Ascidians of Australia. I. Stolidobranchiata and Phlbobranchiata. Australian Journal Marine and Freshwater Research 3: 206-333.

KOTT P (1976) Ascidian fauna of Western Port Bay, Victoria, and a comparison with that of Port Phillip Bay. Memoirs of the National Museum of Victoria 37: 53-95.

LEONARD GH, MD BERTNESS \& PO YUND (1999) Crab predation, waterborne cues, and inducible defenses in the blue mussel, Mytilus edulis. Ecology 80: 1-14.

LEWONTIN RC (1979) La base genética de la evolución. Ediciones Omega, Barcelona, España. 328 pp.

LIVELY CM (1986) Predator-induced shell dimorphism in the acorn barnacle Chthamalus anisopoma. Evolution 40: 232-242.

NEI (1978) Estimation of average heterozygosity and genetic distance from a small number of individuals. Genetics 89: 583-590.

PACHECO CJ \& JC CASTILLA (2000) Ecología trófica de los ostreros Haematopus palliatus pitanay (Murphy 1925) y Haematopus ater (Vieillot et Oudart 1825) en mantos del tunicado Pyura praeputialis (Heller 1878) en la Bahía de Antofagasta, Chile. Revista Chilena de Historia Natural 73: 533-542.

PACKARD GC \& TJ BOARMAN (1988) The misuse of ratios, indices and percentages in ecophysiological research. Physiological Zoology 61: 1-9.

PAINE RT \& TH SUCHANEK (1983) Convergence of ecological processes between independently evolved competitive dominants: a tunicate-mussel comparison. Evolution 37: 821-831

PRESTON JS, CI REVIE, FJ ORR \& D ROBERTS (1996) A comparison of the strengths of gastropod shells with forces generated by potential crab predators. Journal of Zoology (London) 238: 181-193.

RAYMOND M \& F ROUSSET (1995a) GENEPOP (ver 1.2): A population genetics software for exact test and ecumenicism. Journal of Heredity 86: 248-249.

RAYMOND M \& F ROUSSET (1995b) An exact test for population differentiation. Evolution 49: 1280-1283.
REIMCHEN TE (1981) Shell size divergence in Littorina marjae and $L$. obtusata and predation by crabs. Canadian Journal of Zoology 60: 687-695.

SARDA F, C BAS, MI ROLDAN, C PLA \& J LLEONART (1998) Enzymatic and morphometric analyses in Mediterranean populations of the rose shrimp, Aristeus anntennatus (Risso, 1816). Journal of Experimental Marine Biology and Ecology 221: 131-144.

SAS INSTITUTE (1996) SAS/STAT user's guide. Release 6.03 edition. SAS Institute, Cary, North Carolina, U.S.A. 549 pp.

SCHEINER SM (1993) Genetics and evolution of phenotypic plasticity. Annual Review of Ecology and Systematics 24: 35-68.

SLATKIN M (1985) Gene flow in natural populations. Annual Review of Ecology and Systematics 16: 393430.

SLATKIN M (1993) Isolation by distance in equilibrium and non-equilibrium populations. Evolution 47:264279.

SLATKIN M \& NH BARTON (1989) A comparison of three indirect methods for estimating average levels of gene flow. Evolution 43: 1349-1368.

SOKAL RR \& FJ ROHLF (1981) Biometry. Second edition. W.H. Freeman and Company, San Francisco, California. 859 pp.

SOLE-CAVA AM \& JP THORPE (1986) Genetic differentiation between morphotypes of the marine sponge Suberites ficus (Demospogiae: Hadromerida). Marine Biology 93: 247-253.

SOLER R, K WARNKE, U SAINT-PAUL \& D BLOHM (2000) Sequence divergence of mitochondrial DNA indicates cryptic biodiversity in Octopus vulgaris and supports the taxonomic distinctiveness of Octopus mimus (Cephalopoda: Octopodidae). Marine Biology 136: 29-35.

STEARNS SC (1989) The evolutionary significance of phenotypic plasticity. Bioscience 39: 436-445.

SUNDBERG P (1988) Microgeographic variation in shell characters of Littorina saxatilis Olivi, a question mainly of size? Biological Journal of the Linnean Society $35: 169-184$.

SWOFFFORD DL \& SELANDER RK (1981) BIOSYS-1: A FORTRAN program for the comprehensive analysis of electrophoretic data in population genetics and systematics. Journal of Heredity 72: 281-283.

THORPE JP (1982) The molecular clock hypothesis: biochemical evolution, genetic differentiation and systematics. Annual Review of Ecology and Systematics 13: 139-168.

VERMEIJ GJ (1978) Biogeography and adaptation. Patterns of marine life. Harvard University Press, Cambridge, Massachusetts. 332 pp.

VIA S \& R LANDE (1985) Genotype-environment interaction and the evolution of phenotypic plasticity. Evolution 39: 505-522.

WAPLES RS (1987) A multispecies approach to the analysis of gene flow in marine shore fishes. Evolution 41: 385-400.

WEIR BS \& CC COCKERHAM (1984) Estimating Fstatistics for the analysis of population structure. Evolution 38: 1358-1370. 
WEST JM, CD HARVELL \& AM WALLS (1993) Morphological plasticity in a gorgonian coral (Briareum asbestinum) over a depth cline. Marine Ecology Progress Series 94: 61-69.

WHITMORE DH (1990) Electrophoretic and isoelectric focusing techniques in fisheries management. CRC Press, Inc., Boca Raton, Florida. 349 pp.
YUND PO (1991) Natural selection on hydroid colony morphology by intraspecific competition. Evolution 45: 1564-1573.

YUND PO (1995) Gene flow via the dispersal of fertilizing sperm in a colonial ascidian (Botryllus schlosseri): the effect of male density. Marine Biology 122: 649654 .

Editor Asociado: M. Gallardo

Recibido el 25 de septiembre de 2001; aceptado el 8 de abril de 2002 